\title{
Overlapped Fingerprint Separation for Fingerprint Authentication
}

\author{
Neha*, P.P. Gundewar** \\ *(Department of Electronics \& Telecommunication MIT, COE, Pune-411038 \\ Email: neha07uiet@gmail.com) \\ ** (Department of Electronics \& Telecommunication MIT, COE, Pune-411038 \\ Email: pooja.gundewar@mitcoe.edu.in)
}

\begin{abstract}
Overlapped fingerprints captured at the crime scene plays significant role as an evidence to capture the criminals. As latent fingerprints are the accidently left skin impressions, so these are found to be with broken ridge composition, overlapped patterns and spoiled minutiae information. The Graphical User Interface (GUI) system is developed by using MATLAB R2015a software. This project also includes the development of standalone program for this system. The main purpose of GUI development is to get the value of real end points and real-branch points of a overlapped fingerprint image. The value of this point is used in fingerprint image matching process to identify the owner of an overlapped fingerprint image. The image enhancement consists of several process such as histogram equalization process, enhancement by Fast Fourier Transform (FFT) factor, and image binarization while minutiae extraction consist of ridge thinning process, region of interest (ROI) extraction, and minutiae extraction process. All processes should be done one by one.
\end{abstract}

Keywords: Fingerprint Classification, Singular point, Feature Extraction, Neural Network, Matlab.

\section{INTRODUCTION}

Fingerprint identification method is well adapted approach in the many forensic, law enforcement and civil applications for the reliable personal authentication. The fingerprint will continue to be the leading biometric trait and many identity management and access control applications will continue to rely on fingerprint recognition because of its proven performance, the existence of large databases and the availability of compact and cheap fingerprint devices. New research problems have accompanied the wide development of fingerprint technology. It includes feature extraction from low quality images, liveness detection and automated latent fingerprint identification, separating overlapped fingerprint or recognizing one of the fingerprint from an overlapped one. Biometrics system refers to the recognition of a person based on their physical or behavioral characteristics. A good biometric system should be easily assessable, unique and secure over period time. It should be exact, quick, robust, secure and inexpensive. The biometric techniques can potentially prevent unauthorized access to or the fraudulent use of ATM, cellular phones, smart cards, desktop PCs, workstations, and computer networks. Fingerprints are the most widely recognized biometric, particularly for criminal applications and forensic applications. Fingerprints have been routinely used as a method for person identification for more than decades.
Fingerprint recognition has wide application prospect in all fields which contain identity authentication. Construction of accurate and reliable, safe and practical Automatic Fingerprint Identification System (AFIS) has become research hotspot.

Fingerprint and its various features such as minutiae, crossover, core, burification, delta, ridge ending, island, pore. [1]

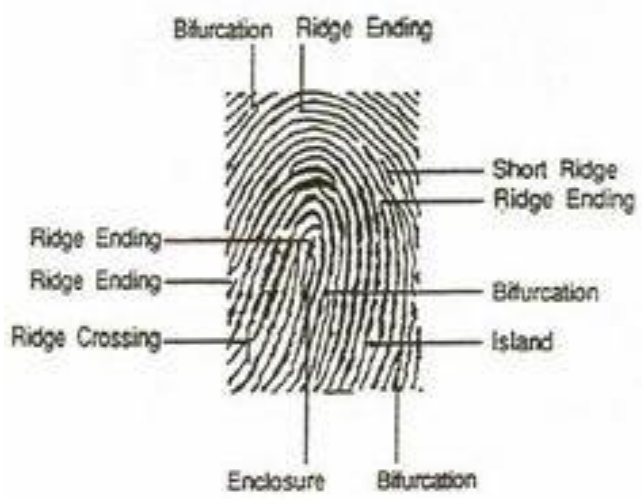

Fig.1 Fingerprint features

\subsection{Overlapped fingerprint}

Overlapped fingerprint image, while difficult to process, are useful forensic evidence for identifying suspects. Available fingerprint matchers, however, cannot accurately match overlapping fingerprints, because they assume that a fingerprint image contains only a single 
fingerprint and hence single orientation field. So algorithms must be developed to separate overlapping latent that will serve as a valuable tool in forensics. In forensics, the matching accuracy of latent is extremely critical.[2]

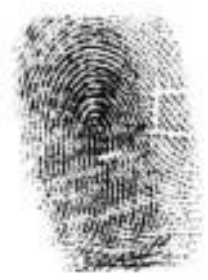

Fig. 2 Overlapped image

Therefore, it is desirable to develop a technique that can automatically separate overlapped fingerprints into their individual fingerprints to improve the matching performance.

\section{PROPOSED SYSTEM}

The proposed system for fingerprint classification involves (i) the extraction of features of given fingerprint image, (ii) Feature Orientation (iii) labeling of each oriented fingerprint in to six class like arch, tented arch, right loop, left loop, twin loop, whorl. The fingerprints have been traditionally classified into categories based on information in the global patterns of ridges. Fingerprint classification provides an important indexing mechanism in a fingerprint database. An accurate and consistent classification can greatly reduce fingerprint matching time from a large database. We present a fingerprint classification algorithm which is able to achieve an accuracy better Efforts in automatic fingerprint classification have been exclusively directed. The fingerprint classification architecture that has shown classification of fingers into the six classes. It consist of user interface to provide interaction for user with system, the system database is the collections of the recorded data and enrolment module and authentication module is present for system input and verification of the fingerprint image given by user.

In the proposed methodology 10 samples are taken which is already stored in the system and in PIC controller of hardware so used. In the hardware, the PIC controller, driver motor for gate to open and close by which the fingerprint is genuine or not can be recognized.

Below figure depicts the flowchart of the complete system:

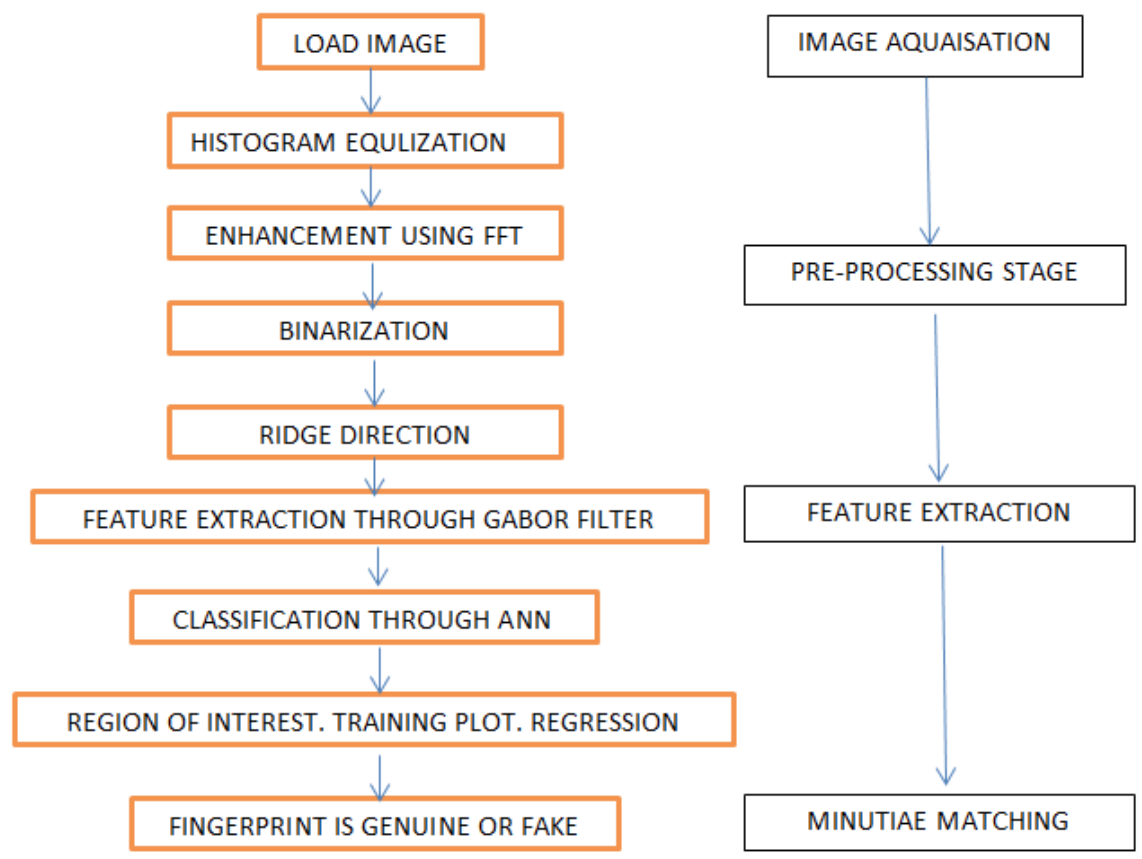

Fig. 3 Proposed methodology

\subsection{Steps involved}

Step1: First we take original fingerprint image and performing image enhancement by using histogram equalization.
Step2: For better enhancement we use histogram equalization image as an input to the FFT algorithm. Step3: The enhanced image is binarized using binarization algorithm. 
Step4: For directional map, orientational flow of estimation is done.

Step5: For extracting the unnecessary part is done with the help of Gabor filter through which a specific point of the overlapped image is extracted so that location of each point can be located.

Step6: After this classification is done by artifical neural network, in which on the basis of region of interest (ROI).

Step7: With the help of the classifier the output on the command window is genuine or fake, depending on the compared data.

\section{CONCLUSION}

The developed overlapped fingerprint separation for fingerprint authentication, clearly displayed technique has enormously enhanced fingerprint image classification accuracy. Simulation results verified that the proposed calculation is precise and powerful.

In addition, Image quality of the overlapped fingerprints used in the current experiments is relatively good. It also compare with the data in the fingerprint classification process, which authenticate that the given sample is genuine or fake. We are in the process of collecting additional latent overlapped fingerprints of various qualities that are lifted using different latent development methods. And also to compare the fingerprints obtained at different ages of a person.

\section{REFERENCES}

[1]. Sankaran, Anu sh, Mayank Vatsa, and Richa Singh. "Automated clarity and quality assessment for latent fingerprints." In Biometrics: Theory, Applications and Systems (BTAS), 2073 IEEE Sixth International Conference on, pp. I - 6.I EEE, 20 I 3.

[2]. Hicklin, R. Austin, JoAnn Buscaglia, and Maria Antonia Roberts. 144 "Assessing the clarity of friction ridge impressions." Forensic science international 226, no. I (2013): 106-11 7.

[3]. Ramaiah, N. Pattabhi, A. Tirupathi Rao, and C. Kri shna Mohan."Enhancements to latent fingerprints in forensic application s. "In 2074 79th International Conference on Digital Signal Processing, pp. 439-443. IEEE, 2014.

[4]. Cao, Kai, Eryun Liu, and Anil K. Jain . "Segmentation and enhancement of latent fingerprints: A coarse to fine rid gestructure dictionary." IEEE transactions on pattern analysis and machine intelligence 36, no. 9 2(014): 1847-1 859. 